\title{
THE STABLE RANK OF CROSSED PRODUCTS OF SECTIONAL C*-ALGEBRAS BY COMPACT LIE GROUPS
}

\author{
ECKART SCHULZ
}

(Communicated by Jonathan M. Rosenberg)

\begin{abstract}
Let $X$ be a second countable, locally compact Hausdorff space, and let a compact Lie group $D$ act on a $C^{*}$-bundle $(E, X)$, the fibres of which are of bounded, finite dimension. Denote by $A$ the $C^{*}$-algebra of sections vanishing at infinity, and by $\hat{\alpha}$ the induced action of $D$ on $A$. The stable ranks of both, the fixed point algebra $A^{\hat{\alpha}}$ and the crossed product algebra $A \times_{\hat{\alpha}} D$ are determined.
\end{abstract}

\section{INTRODUCTION}

In this paper, we study the stable rank of fixed point algebras and $\mathrm{C}^{*}$-crossed products arising from actions of compact Lie groups on commutative or "nearly" commutative $\mathrm{C}^{*}$-algebras. In particular, let $(E, X)$ be a $\mathrm{C}^{*}$-bundle over the second countable, locally compact Hausdorff space $X$, and assume that all fibres $E_{x}$ are finite dimensional, $\operatorname{dim}\left(E_{x}\right) \leq M$ for some fixed $M$ and all $x \in X$. Let $D$ be a compact group acting on $(E, X)$, that is, there exist actions $\alpha_{d}$ and $\varphi_{d}$ of $D$ on $E$ and $X$ respectively, such that the following diagram

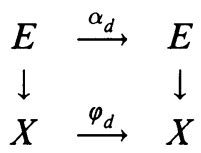

commutes, and $\alpha_{d}$ is a $C^{*}$-isomorphism on fibres; call such a bundle a $D-C^{*}$ bundle. Set $\Gamma_{o}(E)=\Gamma_{o}(E, X)$, the $C^{*}$-algebra of continuous sections vanishing at infinity, and let $\Gamma_{o}^{D}(E)=\left\{e \in \Gamma_{o}(E): e\left(\varphi_{d}(x)\right)=\alpha_{d}(e(x))\right.$ for all $d \in$ $D, x \in X\}$, the algebra of equivariant sections. There is an induced action $\hat{\alpha}$ of $D$ on $\Gamma_{o}(E)$ given by $\left[\hat{\alpha}_{d}(e)\right](x)=\alpha_{d}\left(e\left(\varphi_{d^{-1}}(x)\right)\right)$, and $\Gamma_{o}^{D}(E)$ is the fixed point algebra of $\Gamma_{o}(E)$ under this action, which by Evans' tilde construction [Ev] can be represented as the algebra $\Gamma_{o}(\widetilde{E})$ of sections of a new $\mathrm{C}^{*}$-bundle $(\widetilde{E}, \widetilde{X})$.

Nistor [Ni] has given an explicit formula for the computation of the stable rank of certain separable type I $\mathrm{C}^{*}$-algebras: If the $\mathrm{C}^{*}$-algebra $A$ has a finite

Received by the editors January 2, 1990 and, in revised form, March 9, 1990.

1980 Mathematics Subject Classification (1985 Revision). Primary 46L05; Secondary 22D25.

Key words and phrases. $\mathrm{C}^{*}$-crossed product, stable rank, $\mathrm{C}^{*}$-bundle. 
composition series $\{0\}=I_{0} \subset I_{1} \subset \cdots \subset I_{n+1}=A$ such that each subquotient is of the form $I_{k+1} / I_{k} \cong c_{o}-\sum_{m \in \mathbf{N}} \Gamma_{o}\left(E_{m}^{k}, X_{m}^{k}\right)$, where each $\left(E_{m}^{k}, X_{m}^{k}\right)$ is a locally trivial homogeneous $\mathrm{C}^{*}$-bundle with fibres isomorphic to $M_{m}(\mathbf{C})$, then

$$
\operatorname{sr}\left(I_{k+1} / I_{k}\right)=\sup _{m}\left\lceil\frac{\operatorname{dim}\left(X_{m}^{k}\right)-1}{2 m}\right\rceil+1 \text { and } \operatorname{sr}(A)=\max _{0 \leq k \leq n} \operatorname{sr}\left(I_{k+1} / I_{k}\right) .
$$

Here $\operatorname{dim}(X)$ denotes the covering dimension of the space $X$, and $\lceil x\rceil$ denotes the least integer $\geq x .(\operatorname{dim}(X)=\infty$ is permitted in an obvious way.)

This, in combination with Evans' results, allows us to determine $\operatorname{sr}\left(\Gamma_{o}^{D}(E)\right)$ for an action of a compact Lie group $D$ on a $C^{*}$-bundle $(E, X)$ as above, and to obtain that $\operatorname{sr} \Gamma_{o}(E) \times_{\hat{\alpha}} D \leq \operatorname{sr} \Gamma_{o}(E)$, provided that $X$ has locally finite orbit structure.

\section{SOME FACTS AND LEMMAS}

It will be useful to review Evans' tilde construction and state some lemmas needed in the main proofs. Given an action of the compact group $D$ on the $C^{*}$-bundle $(E, X)$ by pairs $\left(\alpha_{d}, \varphi_{d}\right)$, let $D_{x}$ denote the stabilizer of $x \in X$, and let $F_{x}$ be the fixed point algebra of the fibre $E_{x}$ under the restriction of $\alpha$ to $D_{x}$. Put $F=\bigcup_{x \in X} F_{x}$ and give it the subspace topology of $E$. Define $\widetilde{X}=X / D$ and $\widetilde{E}=F / D$ and give them the quotient topologies. Then $(\widetilde{E}, \widetilde{X})$ is a $C^{*}$-bundle, the map $F \rightarrow \widetilde{E}$ is an isomorphism when restricted to a fibre $F_{x}$, and the quotient map $\pi: X \rightarrow \widetilde{X}$ is open and closed. If, as will be assumed throughout this paper, $X$ is locally compact, second countable and Hausdorff, then $\widetilde{X}$ inherits these properties, and both spaces are $\sigma$-compact, separable and metrizable. If $Y$ is a locally compact $D$-invariant subset of $X$, then $\widetilde{E}_{\mid \widetilde{Y}} \cong \widetilde{E_{\mid Y}}$.

The following lemma clarifies part (b) of Nistor's Theorem 7 in our $C^{*}$ bundle case.

Lemma 2.1. Let $\left\{X_{j}\right\}_{j=1}^{t}$ be a partition of $X$ such that $\bigcup_{l \geq j} X_{l}$ is closed in $X$ for all $j$. Then,

$$
\operatorname{sr}\left(\Gamma_{o}(E)\right)=\max _{j} \operatorname{sr}\left(\Gamma_{o}\left(E_{\mid X_{j}}\right)\right) .
$$

Proof. Proceeding by induction, we may assume that $t=2$. Pick a sequence $\left\{F_{n}\right\}$ of compact subsets of $X_{1}$ such that $F_{n} \subset \stackrel{\circ}{F}_{n+1}$ and $X_{1}=\bigcup_{n=1}^{\infty} F_{n}$. Then by [She, Proposition 3.15],

$$
\begin{aligned}
\operatorname{sr}\left(\Gamma_{o}(E)\right) & \leq \sup _{n}\left\{\operatorname{sr}\left(\Gamma_{o}\left(E_{\mid X_{2}}\right)\right), \operatorname{sr}\left(\Gamma_{o}\left(E_{\mid F_{n}}\right)\right)\right\} \\
& \leq \max \left\{\operatorname{sr}\left(\Gamma_{o}\left(E_{\mid X_{2}}\right)\right), \operatorname{sr}\left(\Gamma_{o}\left(E_{\left|X_{1}\right|}\right)\right)\right\} \leq \operatorname{sr}\left(\Gamma_{o}(E)\right) .
\end{aligned}
$$

Recall that as a consequence of [Fe, Theorem 3.1], homogeneous $\mathrm{C}^{*}$-bundles with finite dimensional fibres are locally trivial. For convenience of notation, let us denote by $M_{\infty}$ the algebra $\mathscr{K}(\mathscr{H})$ of compact operators on the infinite dimensional, separable Hilbert space $\mathscr{H}$. 
Lemma 2.2. Let $(E, X)$ be a locally trivial $D-C^{*}$-bundle with $E_{x} \subset M_{\infty}$ for all $x \in X$. Let $\left\{\left[A_{i}\right]\right\}_{i=1}^{\infty}$ denote the collection of isomorphism classes of fibres of $E$ and set $X_{i}=\left\{x \in X: E_{x} \in\left[A_{i}\right]\right\}$. Then,

(i) $\left\{X_{i}\right\}_{i=1}^{\infty}$ is a collection of mutually disjoint clopen subsets covering $X$.

(ii) The bundle $E_{\mid X_{i}}$ obtained by restriction is D-invariant and

$$
\Gamma_{o}^{D}(E) \cong c_{o}-\sum_{i=1}^{\infty} \Gamma_{o}^{D}\left(E_{\mid X_{i}}\right)
$$

Proof. (i) is obvious by local triviality.

(ii) By (i), $E$ decomposes into the disjoint union of clopen sets, $E=$ $\bigcup_{i=1}^{\infty} E_{\mid X_{i}}$. Because $D$ acts as an isomorphism on fibres, each $E_{\mid X_{i}}$ is $D$-invariant. One checks easily that the map

$$
\phi:\left\{\begin{array}{l}
\Gamma_{o}^{D}(E) \rightarrow c_{o}-\sum_{i=1}^{\infty} \Gamma_{o}^{D}\left(E_{\mid X_{i}}\right) \\
e \rightarrow \sum_{i=1}^{\infty} e_{\mid X_{i}}, \quad e \in \Gamma_{o}^{D}(E)
\end{array}\right.
$$

is a $\mathrm{C}^{*}$-isomorphism.

Now let $A$ be a fixed $C^{*}$-algebra of the form $A=c_{o}-\sum_{k \in \mathbf{N}} n_{k} \circ M_{k}$, where $0 \leq n_{k} \leq \aleph_{0}$ and $n_{k} \circ M_{k}=c_{o}-\sum_{i=1}^{n_{k}} M_{k}$.

Proposition 2.3. Let $(E, X)$ be a locally trivial, homogeneous $D-C^{*}$-bundle whose fibres are isomorphic to $A$. Then there exist (locally compact, second countable Hausdorff) spaces $Y^{k}$ and $D-C^{*}$-bundles $\left(F^{k}, Y^{k}\right)$ such that

(i) each $F^{k}$ is homogeneous, locally trivial with fibres $M_{k}$,

(ii) $\Gamma_{o}(E)=c_{o}-\sum_{k} \Gamma_{o}\left(F^{k}\right)$ and $\Gamma_{o}^{D}(E)=c_{o}-\sum_{k} \Gamma_{o}^{D}\left(F^{k}\right)$,

(iii) $\operatorname{dim}\left(Y^{k}\right)=\operatorname{dim}(X)$ for all $k$.

Proof. Let $\bar{F}_{x}^{k}$ be the subalgebra of the fibre $E_{x}$ isomorphic to $n_{k} \circ M_{k}$, and set $\bar{F}^{k}=\bigcup_{x \in X} \bar{F}_{x}^{k}$. Then $\left(\bar{F}^{k}, X\right)$ is a locally trivial subbundle of $(E, X)$, and it is $D$-invariant as $D$ acts by isomorphism on fibres.

Let $e_{x}^{k}$ denote the projection of $E_{x}$ onto $\bar{F}_{x}^{k}$. A partition of unity argument and [Le, Lemma 2] show that $e^{k}: x \rightarrow e_{x}^{k}$ is a section in the multiplier algebra $\mathscr{M}\left(\Gamma_{o}(E)\right)$ which also is $D$-invariant under the extension of $\hat{\alpha}$ to $\mathscr{M}\left(\Gamma_{o}(E)\right)$. Then, $\Gamma_{o}\left(\bar{F}^{k}\right)=e^{k} \Gamma_{o}(E), \quad \Gamma_{o}^{D}\left(\bar{F}^{k}\right)=e^{k} \Gamma_{o}^{D}(E)$, and since $\left\{e^{k}\right\}_{k=1}^{\infty}$ is a decomposition of the identity of $\mathscr{M}\left(\Gamma_{o}(E)\right)$ into mutually orthogonal central projections, one obtains that $\Gamma_{o}(E)=c_{o}-\sum_{k} \Gamma_{o}\left(\bar{F}^{k}\right)$ and $\Gamma_{o}^{D}(E)=c_{o}-\sum_{k} \Gamma_{o}^{D}\left(\bar{F}^{k}\right)$.

Now set $Y^{k}=\Gamma_{o}\left(\bar{F}^{k}\right)^{\wedge}$. By [Fe, Theorem 1.1], $Y^{k}$ can be identified as a set with $X \times\left(1, \ldots, n_{k}\right)$ for all $k$. Pick an open cover $\left\{U_{i}\right\}_{i=1}^{\infty}$ of $X$ such that $\bar{U}_{i}$ is compact and $E_{\mid U_{i}}$ is trivial. Then $\Gamma_{o}\left(\bar{F}_{\mid U_{i}}^{k}\right)^{\wedge}$ is homeomorphic to 
the product space $U_{i} \times\left(1, \ldots, n_{k}\right)$, and because $\Gamma_{o}\left(\bar{F}_{\mid U_{i}}^{k}\right)$ is a closed ideal in $\Gamma_{o}\left(F^{k}\right), U_{i} \times\left(1, \ldots, n_{k}\right)$ is open in $Y^{k}$. Thus, $\left\{U_{i} \times\{s\}\right\}_{i=1, s=1}^{\infty n_{k}}$ is an open cover of $Y^{k}$, in particular $Y^{k}$ is locally compact, second countable Hausdorff. By the sum theorem, $\operatorname{dim}\left(Y^{k}\right)=\sup _{i, s} \operatorname{dim}\left(\bar{U}_{i} \times\{s\}\right)=\sup _{i} \operatorname{dim}\left(U_{i}\right)=\operatorname{dim}(X)$. Now it is known ([Fe, Theorem 3.2]) that the algebra of sections $\Gamma_{o}\left(F^{k}\right)$ of the homogeneous bundle $\left(F^{k}, X\right)$ is isomorphic to the algebra $\Gamma_{o}\left(F^{k}\right)$ of sections of a bundle $\left(F^{k}, Y^{k}\right)$ with fibres $M_{k}$. Since this isomorphism maps $\Gamma_{o}\left(\bar{F}_{\mid U_{i}}^{k}\right) \cong$ $C_{o}\left(U_{i}\right) \otimes\left(c_{o}-\sum_{i=1}^{n_{k}} M_{k}\right) \cong C_{o}\left(U_{i} \times\left(1, \ldots, n_{k}\right)\right) \otimes M_{k}$ onto $\Gamma_{o}\left(F_{\mid U_{i} \times\left(1, \ldots, n_{k}\right)}^{k}\right)$, the bundle $\left(F^{k}, Y^{k}\right)$ is locally trivial. Here $C_{o}(X)$ denotes the set of continuous functions on a locally compact space $X$ that vanish at infinity.

Suppose now, the bundle automorphism $\left(\alpha_{d}, \varphi_{d}\right)$ maps the element $e_{i}$ in the $i$ th component of $F_{x}^{k}$ to the element $f_{j}$ in the $j$ th component of $F_{\varphi_{d}(x)}^{k}$. Define

$$
\left\{\begin{array}{l}
\gamma_{d}: e_{i} \in M_{k} \rightarrow f_{j} \in M_{k} \\
\psi_{d}:(x, i) \in Y^{k} \rightarrow\left(\varphi_{d}(x), j\right) \in Y^{k} .
\end{array}\right.
$$

One easily checks that $\left(\gamma_{d}, \psi_{d}\right)$ defines an action of $D$ on $\left(F^{k}, Y^{k}\right)$ and that the above isomorphism maps $\Gamma_{o}^{D}\left(\bar{F}^{k}\right)$ onto $\Gamma_{o}^{D}\left(F^{k}\right)$.

Let $D$ be a second countable compact group, $L$ a closed subgroup, and denote by $m_{D}$ and $m_{L}$ the normalized Haar measures on $D$ and $L$, respectively. If $\pi: D \rightarrow D / L$ denotes the quotient map, then we have an invariant regular Borel measure $\mu$ on $D / L$ defined by $\mu(Q)=m_{D}\left(\pi^{-1}(Q)\right)$ on Borel sets $Q \subset D / L$. Fix a Borel cross-section $\gamma: D / L \rightarrow D$. Then by the uniqueness of the Haar measure, $\int_{D} f(d) d m_{D}(d)=\int_{D / L} \int_{L} f(\gamma(q) l) d m_{L}(l) d \mu(q)$ for all $f \in C(D)$, the set of continuous functions on $D$. (See [Lo], $\S 33$.)

Lemma 2.4. The $\mathrm{C}^{*}$-dynamical systems

$$
\left(\mathscr{K}\left(L^{2}(D)\right), \operatorname{Ad} \rho(D)_{\mid L}, L\right) \text { and }\left(\mathscr{K}\left(L^{2}(L)\right) \otimes \mathscr{K}\left(L^{2}(D / L)\right), \operatorname{Ad} \widetilde{\rho}(L) \otimes i d, L\right)
$$

are covariantly isomorphic. Here $\rho$ and $\tilde{\rho}$ denote the right regular representations of $D$ and $L$, respectively.

Proof. First identify $L^{2}(D / L) \otimes L^{2}(L)$ with $L^{2}\left(D / L, L^{2}(L)\right)$. On elementary tensors $p \otimes f \in L^{2}(D / L) \otimes L^{2}(L)$, the identification is given by $[(p \otimes f)(q)](l)=$ $p(q) f(l)$ for $q \in D / L, l \in L$. Now define

$$
U: L^{2}(D) \rightarrow L^{2}\left(D / L, L^{2}(L)\right)
$$

by

$$
[(U f)(q)](l)=f(\gamma(q) l)
$$

for $f \in L^{2}(D)$. As

$$
\|U f\|^{2}=\int_{D / L} \int_{L}|f(\gamma(q) l)|^{2} d m_{L}(l) d \mu(q)=\int_{D}|f(d)|^{2} d m_{D}(d)=\|f\|^{2},
$$


it follows that $U$ is an isomorphism of the two Hilbert spaces.

Note that for $q \in D / L$ and $d, l \in L$,

$$
\left[\left(U \rho_{d} U^{*}\right)(p \otimes f)\right](q)(l)=(p \otimes f)(q)(l d)=p(q) f(l d)=\left(p \otimes \tilde{\rho}_{d} f\right)(q)(l),
$$

so that by linearity and continuity,

$$
U \rho_{d} U^{*}=i d \otimes \tilde{\rho}_{d}
$$

and thus,

$$
\operatorname{Ad} U \operatorname{Ad} \rho_{d} \operatorname{Ad} U^{*}=i d \otimes \operatorname{Ad} \tilde{\rho}_{d} .
$$

Two actions $\alpha$ and $\beta$ of a locally compact group $D$ on a $C^{*}$-algabra $A$ are exterior equivalent, if there exists a strictly continuous map $d \rightarrow u_{d}$ of $D$ into the unitary group $\mathscr{U}(M(A))$ such that

$$
\alpha_{d}(a)=u_{d} \beta_{d}(a) u_{d}^{*} \quad d \in D, a \in A
$$

and

$$
u_{c d}=u_{c} \beta_{c}\left(u_{d}\right) \quad c, d \in D,
$$

where $\beta$ is extended to the multiplier algebra $\mathscr{M}(A)$ in a unique way. Exterior equivalent actions give rise to isomorphic crossed products.

Given a separable Hilbert space $\mathscr{H}$, identify $C_{o}(X, \mathscr{K}(\mathscr{H}))$ with the algebra $\Gamma_{o}(\mathscr{K}(\mathscr{H}) \times X)$ of sections of the trivial bundle. Its multiplier algebra is isomorphic to the set $C^{b}(X, \mathscr{B}(\mathscr{H}))$ of bounded, strictly (and hence strong-*) continuous functions from $X$ to $\mathscr{B}(\mathscr{H})$. There is a one-to-one correspondence between bundle actions of the form $(\alpha, i d)$ and center-fixing actions $\hat{\alpha}$ on $C_{o}(X, \mathscr{K}(\mathscr{H}))$. Denote by $\alpha^{x}$ the restriction of $\alpha$ to the fibre $\mathscr{K}(\mathscr{H}) \times\{x\}$.

The following result is probably well known, although I have found no reference.

Proposition 2.5. Let $\hat{\alpha}$ and $\hat{\beta}$ be center-fixing actions of a second countable compact group $D$ on $C_{o}(X, \mathscr{H}(\mathscr{H}))$, and assume that for some $x_{o} \in X$, the actions $\alpha^{x_{o}}$ and $\beta^{x_{o}}$ are exterior equivalent. Then there exists a neighborhood $V$ of $x_{o}$ such that the restrictions of $\hat{\alpha}$ and $\hat{\beta}$ to $C_{o}(V, \mathscr{K}(\mathscr{H}))$ are exterior equivalent.

Proof. As in the proof of [PhR, Proposition 1.3], there exist a compact neighborhood $V$ of $x_{o}$ and Borel maps $v, w: D \rightarrow C^{b}(V, \mathscr{B}(\mathscr{H}))$ such that

$$
\hat{\alpha}_{d}(a)=v_{d} a v_{d}^{*} \quad \text { and } \quad \hat{\beta}_{d}(a)=w_{d} a w_{d}^{*}
$$

for $a \in C(V, \mathscr{K}(\mathscr{H}))$ and $d \in D$. Let $u: D \rightarrow \mathscr{U}(\mathscr{H})$ be the unitary 1-cocycle implementing the exterior equivalence of $\alpha^{x_{o}}$ and $\beta^{x_{o}}$. Since $u_{d} w_{d}\left(x_{o}\right)$ and $v_{d}\left(x_{o}\right)$ differ only by a scalar, we may assume w.l.o.g. that $v_{d}\left(x_{o}\right)=u_{d} w_{d}\left(x_{o}\right)$. Then

$$
\rho(c, d)=v_{c} v_{d} v_{c d}^{*} \quad \text { and } \quad \psi(c, d)=w_{c} w_{d} w_{c d}^{*}
$$

define Borel cocycles $D \times D \rightarrow C(V, \mathbf{T})$ such that

$$
\begin{aligned}
\rho(c, d)\left(x_{o}\right) & =u_{c} w_{c}\left(x_{o}\right) u_{d} w_{d}\left(x_{o}\right) w_{c d}^{*}\left(x_{o}\right) u_{c d}^{*} \\
& =u_{c d} w_{c}\left(x_{o}\right) w_{d}\left(x_{o}\right) w_{c d}^{*}\left(x_{o}\right) u_{c d}^{*}=\psi(c, d)\left(x_{o}\right)
\end{aligned}
$$


so that the 2-cocycle $\sigma=\rho \bar{\psi}$ satisfies $\sigma(c, d)\left(x_{o}\right)=1$ for all $c, d \in D$. The map $y \in V \rightarrow \sigma(\cdot, \cdot)(y)$ gives a family of cocycles $D \times D \rightarrow \mathbf{T}$ varying continuously in $y$, and as $H^{2}(D, \mathbf{T})$ is discrete by compactness of $D, \sigma(\cdot, \cdot)(y)$ is a coboundary for $y$ close to $x_{o}$. Cutting down on $V$ if necessary, we may by [Ro, Theorem 2.1] assume that $\sigma$ is a coboundary, that is, there exists a Borel map $\lambda: D \rightarrow C(V, T)$ such that $\sigma(c, d)=\lambda(c) \lambda(d) \overline{\lambda(c d)}$ for almost all $c, d \in D$. Set $z_{d}=\overline{\lambda(d)} v_{d} w_{d}^{*}$. Then

$$
z_{d} \hat{\beta}_{d}(a) z_{d}^{*}=v_{d} w_{d}^{*} \hat{\beta}_{d}(a) w_{d} v_{d}^{*}=v_{d} a v_{d}^{*}=\hat{\alpha}_{d}(a)
$$

and

$$
\begin{aligned}
z_{c d} & =\overline{\lambda(c d)} v_{c d} w_{c d}^{*}=\sigma(c, d) \overline{\lambda(c) \lambda(d) \rho(c, d)} v_{c} v_{d} \psi(c, d) w_{d}^{*} w_{c}^{*} \\
& =z_{c} w_{c} \overline{\lambda(d)} v_{d} w_{d}^{*} w_{c}^{*}=z_{c} \hat{\beta}_{c}\left(z_{d}\right)
\end{aligned}
$$

for almost all $c, d \in D$. Finally, by [Mo, Theorem 3], we may assume that $d \rightarrow z_{d}$ is strictly continuous, so that (1) and (2) hold for all $c, d \in D$.

Rieffel [Ri] has shown that given a compact $\mathrm{C}^{*}$-dynamical system $(A, D, \alpha)$, the crossed product $A \times{ }_{\alpha} D$ is isomorphic to the fixed point algebra $\left[A \otimes \mathscr{K}\left(L^{2}(D)\right)\right]^{\alpha \otimes \operatorname{Ad} \rho}$, and he has given an identification of $\mathscr{K}\left(L^{2}(D)\right)$ with $C(D) \times_{\lambda} D$ under which the $D$-action Ad $\rho$ on $\mathscr{K}\left(L^{2}(D)\right)$ corresponds to the action $\gamma$ defined on the dense subset $C(D \times D) \cong C(D, C(D))$ of $C(D) \times{ }_{\lambda} D$ by $\left[\gamma_{s}(f)\right](c, d)=f(c, d s)$ for $f \in C(D \times D), s, c, d \in D$. Here $\lambda$ and $\rho$ denote the left and right regular representations of $D$ on $L^{2}(D)$, and $\operatorname{Ad} \rho_{d}(T)=$ $\rho_{d} T \rho_{d^{-1}}$ for all $T \in \mathscr{K}\left(L^{2}(D)\right)$ and $d \in D$.

Given an action $\left(\alpha_{d}, \varphi_{d}\right)$ of the compact group $D$ on the $\mathrm{C}^{*}$-bundle $(E, X)$, let $(\bar{E}, X)$ denote the tensor product bundle, that is the bundle with fibres $\bar{E}_{x}=E_{x} \otimes \mathscr{K}\left(L^{2}(D)\right)$, and give it an action $\left(\bar{\alpha}_{d}, \varphi_{d}\right)$ by

(3) $\bar{\alpha}_{d}: a \otimes T \in E_{x} \otimes \mathscr{K}\left(L^{2}(D)\right) \rightarrow\left[\alpha_{d} \otimes \operatorname{Ad} \rho_{d}\right](a \otimes T) \in E_{\varphi_{d}(x)} \otimes \mathscr{K}\left(L^{2}(D)\right)$.

Then $\Gamma_{o}(E) \otimes \mathscr{K}\left(L^{2}(D)\right) \cong \Gamma_{o}(\bar{E})$ and $\Gamma_{o}(E) \times_{\hat{\alpha}} D \cong\left[\Gamma_{o}(E) \otimes \mathscr{K}\left(L^{2}(D)\right)\right]^{\hat{\alpha} \otimes \operatorname{Ad} \rho}$ $\cong \Gamma_{o}^{D}(\bar{E})$.

Corollary 2.6. Let the second countable compact group $D$ act on the trivial bundle $(E, X)=\mathscr{K}(\mathscr{H}) \times X$ by actions $\left(\alpha_{d}\right.$, id $)$. Then, given $x \in X$, there exists a neighborhood $V$ of $x$ such that $\widetilde{E_{\mid V}}$ is trivial. In particular, $\Gamma_{o}\left(E_{\mid V}\right) \times_{\hat{\alpha}} D \cong C_{o}(V) \otimes\left(\mathscr{K}(\mathscr{H}) \times_{\alpha^{x}} D\right)$.

Proof. Define an action $(\beta, i d)$ of $D$ on $(E, X)$ by $\beta_{d}^{y}=\alpha_{d}^{x}$ for $y \in X$ and pick $V$ as in the last proposition with $x_{o}=x$.

Define a bundle isomorphism $(\phi, i d): \bar{E}_{\mid V} \rightarrow \bar{E}_{\mid V}$ by

$$
\phi(a)(c, d)=z_{d^{-1}}(y) a(c, d) z_{d^{-1} c}(y)^{*}
$$


for $a$ an element of the dense subset $C\left(D \times D, E_{y}\right)$ of the fibre $\bar{E}_{y}$ and $c, d \in D$. Then,

$$
\begin{aligned}
{\left[\left(\alpha_{s} \otimes \gamma_{s}\right) \phi(a)\right](c, d) } & =\alpha_{s}[(\phi(a))(c, d s)]=\alpha_{s}\left(z_{(d s)^{-1}}(y) a(c, d s) z_{(d s)^{-1} c}(y)^{*}\right) \\
& =z_{s}(y) \beta_{s}\left(z_{(d s)^{-1}}(y) a(c, d s) z_{(d s)^{-1} c}(y)^{*}\right) z_{s}(y)^{*} \\
& =z_{d^{-1}}(y) \beta_{s}(a(c, d s)) z_{d^{-1} c}(y)^{*} \\
& =z_{d^{-1}}(y)\left[\left(\left(\beta_{s} \otimes \gamma_{s}\right)(a)\right)(c, d)\right] z_{d^{-1} c}(y)^{*} \\
& =\varphi\left(\left(\beta_{s} \otimes \gamma_{s}\right)(a)\right)(c, d) .
\end{aligned}
$$

So $\phi$ intertwines the two bundle actions, and thus restricts to an isomorphism of the tilde bundles. As the tilde bundle associated with $\left(\beta_{s} \otimes \gamma_{s}\right)$ is trivial, the statement follows.

\section{COMPACT GROUP ACTIONS}

Throughout this section, $(E, X)$ will denote a $C^{*}$-bundle whose fibres are of bounded finite dimension, that is, there exists an integer $M$ such that $\operatorname{dim}\left(E_{x}\right)$ $\leq M$ for all $x \in X$.

Let us first determine the stable rank of $\Gamma_{o}(E)$ : Denote by $\left[A_{1}\right], \ldots,\left[A_{r}\right]$ the collection of isomorphism classes of fibres of $E$, ordered such that $i<k \Rightarrow$ $\operatorname{dim}\left(A_{i}\right) \geq \operatorname{dim}\left(A_{k}\right)(1 \leq i, k \leq r)$. Each $A_{k}$ is a sum of matrix algebras,

$$
A_{k}=\sum_{q=N_{k}}^{M_{k}} n_{k}^{q} \circ M_{q} \quad\left(0 \leq n_{k}^{q}<\infty\right),
$$

where $M_{N_{k}}$ denotes the smallest matrix component of $A_{k}$. Set $X_{i}=\{x \in X$ : $\left.E_{x} \in\left[A_{i}\right]\right\}$. Because of [Fe, Theorem 3.1], $\bigcup_{i>k} X_{i}$ is closed in $X$ for all $k$. Setting $I_{k}=\left\{f \in \Gamma_{o}(E): f\right.$ vanishes on $\bigcup_{i \geq k} X_{i} \cong \Gamma_{o}\left(E_{\mid X-\cup_{i \geq k} X_{i}}\right)$, we obtain a decomposition series of closed ideals

$$
\{0\}=I_{1} \subset I_{2} \subset \cdots \subset I_{r} \subset I_{r+1}=\Gamma_{o}(E)
$$

with subquotients

$$
I_{k+1} / I_{k} \cong \Gamma_{o}\left(E_{\mid X_{k}}\right) .
$$

By Proposition 2.3, each of $I_{k+1} / I_{k}$ satisfies condition (A) of Nistor's Theorem 7, so that

$$
\operatorname{sr}\left(\Gamma_{o}(E)\right)=\max _{1 \leq k \leq r}\left\lceil\frac{\operatorname{dim}\left(X_{k}\right)-1}{2 N_{k}}\right\rceil+1 .
$$

Note also that

$$
\operatorname{sr}\left(C_{o}(X)\right)=\max _{1 \leq k \leq r}\left\lceil\frac{\operatorname{dim}\left(X_{k}\right)-1}{2}\right\rceil+1
$$

by the same theorem.

Now let $D$ be a compact group acting on the $\mathrm{C}^{*}$-bundle $(E, X)$. Let $\pi: X \rightarrow$ $\tilde{X}=X / D$ denote the quotient map. We say that $\pi$ has local cross-sections if 
given $\tilde{x} \in \tilde{X}$ there exists a neighborhood $U$ of $\tilde{x}$ such that $\pi_{\mid \pi^{-1}(U)}$ has a crosssection. Note that this implies that $\operatorname{dim}(\tilde{X}) \leq \operatorname{dim}(X)$ by the sum theorem. If $D$ is compact Lie, then always $\operatorname{dim}(\widetilde{X}) \leq \operatorname{dim}(X)$ (see [Pa], Theorem 3.16). In both cases, $\operatorname{dim}(X)-t \leq \operatorname{dim}(\widetilde{X})$ by a theorem on dimension lowering mappings ([En],1.12.4), where $t$ denotes the largest of the dimensions of the orbits.

Proposition 3.1. If there exist local cross-sections or if $D$ is a Lie group, then

$$
\sup _{1 \leq k \leq r}\left\{\operatorname{sr}\left(C_{o}\left(\widetilde{X_{k}}\right) \otimes M_{N_{k}}\right)\right\} \leq \operatorname{sr}\left(\Gamma_{o}^{D}(E)\right) \leq \operatorname{sr}\left(C_{o}(\widetilde{X})\right) \leq \operatorname{sr}\left(C_{o}(X)\right) .
$$

Proof. Since the ideals $I_{k}$ in (5) are $D$-invariant, $\Gamma_{o}^{D}(E) \cong \Gamma_{o}(\widetilde{E})$ has a composition series whose subquotients are isomorphic to $\Gamma_{o}^{D}\left(E_{\mid X_{k}}\right) \cong \Gamma_{o}\left(\widetilde{E}_{\mid \widetilde{X}_{k}}\right)$. An application of the above process shows that $\Gamma_{o}\left(\widetilde{E}_{\mid \widetilde{X}_{k}}\right)$ satisfies Nistor's condition A) and allows us to compute $\operatorname{sr}\left(\Gamma_{o}\left(\widetilde{E}_{\mid \widetilde{x}_{k}}\right)\right)$. Since the fibres of $\widetilde{E}_{\mid \widetilde{X}_{k}}$ are subalgebras of $A_{k}(1 \leq k \leq r)$, we obtain the following estimate:

$$
\left\lceil\frac{\operatorname{dim}\left(\widetilde{X}_{k}\right)-1}{2 N_{k}}\right\rceil+1 \leq \operatorname{sr}\left(\Gamma_{o}\left(\widetilde{E}_{\mid \widetilde{X}_{k}}\right)\right) \leq\left\lceil\frac{\operatorname{dim}\left(\widetilde{X}_{k}\right)-1}{2}\right\rceil+1
$$

and therefore,

$$
\begin{aligned}
& \sup _{1 \leq k \leq r}\left\lceil\frac{\operatorname{dim}\left(\widetilde{X_{k}}\right)-1}{2 N_{k}}\right\rceil+1 \leq \operatorname{sr}\left(\Gamma_{o}(\widetilde{E})\right) \\
& \quad \leq \sup _{1 \leq k \leq r}\left\lceil\frac{\operatorname{dim}\left(\widetilde{X_{k}}\right)-1}{2}\right\rceil+1=\operatorname{sr}\left(C_{o}(\widetilde{X})\right) .
\end{aligned}
$$

Remark. Note that $\operatorname{sr}\left(\Gamma_{o}^{D}(E)\right)$ may be larger or smaller than $\operatorname{sr}\left(\Gamma_{o}(E)\right)$.

(1) If the $r$-torus $\mathbf{T}^{r}(r \geq 4)$ acts on the trivial bundle $E=\mathbf{C} \times \mathbf{T}^{r}$ by $t:(a, x) \rightarrow(a, t x)\left(a \in \mathbf{C}, t, x \in \mathbf{T}^{r}\right)$, then $\Gamma_{o}(\widetilde{E}) \cong \mathbf{C}$ and $\Gamma_{o}(E) \cong C\left(\mathbf{T}^{r}\right)$, so that $1=\operatorname{sr}\left(\Gamma_{o}^{D}(E)\right)<\operatorname{sr}\left(\Gamma_{o}(E)\right)=\left\lceil\frac{r-1}{2}\right\rceil+1$.

(2) If the $r$-torus acts on $E=M_{r} \times \mathbf{T}^{r}$ by

$$
t=\left(t_{1}, \ldots, t_{r}\right):(a, x) \rightarrow\left(\left(\sum t_{i} e_{i i}\right) a\left(\sum \bar{t}_{j} e_{j j}\right), x\right),
$$

then $\Gamma_{o}(E) \cong C\left(\mathbf{T}^{r}, M_{r}\right)$ while $\Gamma_{o}(\widetilde{E}) \cong C\left(\mathbf{T}^{r}, \mathbf{C}^{r}\right)$, so that $\left\lceil\frac{r-1}{2}\right\rceil+1=$ $\operatorname{sr}\left(\Gamma_{o}^{D}(E)\right)>\operatorname{sr}\left(\Gamma_{o}(E)\right)=2$.

However, we have the following, which always holds for finite groups:

Corollary 3.2. If all orbits are finite, then $\operatorname{sr}\left(\Gamma_{o}(E)\right) \leq \operatorname{sr}\left(\Gamma_{o}^{D}(E)\right) \leq \operatorname{sr}\left(C_{o}(X)\right)$. Proof. By [En], Theorem 1.12.8, $\operatorname{dim}\left(\widetilde{X_{k}}\right)=\operatorname{dim}\left(X_{k}\right)$ for all $k$. The assertion then follows from (7) and (9).

Corollary 3.3. Let $G$ be a second countable, locally compact group and $A$ an abelian normal subgroup of $G$ of finite index, say $D=G / A$ has order $n$. Then,

$$
\operatorname{sr}\left(C^{*}(A) \otimes M_{n}\right) \leq \operatorname{sr}\left(C^{*}(G)\right) \leq \operatorname{sr}\left(C^{*}(A)\right) .
$$


Proof. Taylor [Ta] has shown that $C^{*}(G) \cong \Gamma_{o}^{D}(E)$, where $D$ acts on the trivial bundle $E=M_{n} \times \hat{A}$. Since $C^{*}(A) \cong C_{o}(\hat{A})$, the statement follows immediately from Proposition 3.1.

We say that $X$ has locally finite orbit structure, if given $x \in X$ there exists an open neighborhood $U$ of $x$ such that the types of orbits touching $U$ are finite in number. By [Bre], IV.1.2, this always happens if $D$ is compact Lie and $X$ is a topological manifold.

Theorem 3.4. Let the compact Lie group $D$ act on $(E, X)$ by actions $\left(\alpha_{d}, \varphi_{d}\right)$, and assume that $X$ has locally finite orbit structure. Then there exist numbers $l, s \in \mathbf{N} \cup\{\infty\}$ such that

$$
\operatorname{sr}\left(C_{o}(\tilde{X}) \otimes M_{l}\right) \leq \operatorname{sr}\left(\Gamma_{o}(E) \times_{\hat{\alpha}} D\right) \leq \operatorname{sr}\left(\Gamma_{o}(E) \otimes M_{s}\right) .
$$

Proof. Let $(\bar{E}, X)$ denote the tensor product bundle with fibres $E_{x} \otimes$ $\mathscr{K}\left(L^{2}(D)\right)$ and $D$-action $\left(\bar{\alpha}_{d}, \varphi_{d}\right)$ as in (3), so that $\Gamma_{o}(E) \times_{\hat{\alpha}} D \cong \Gamma_{o}^{D}(\bar{E})$.

Assume first that $X$ is compact, so that it has finite orbit structure globally, and that $E$ is homogeneous with fibres isomorphic to the matrix algebra $M_{n}$. Let $\left[D_{1}\right], \ldots,\left[D_{t}\right]$ denote the conjugacy classes of stabilizer subgroups ordered such that $\exists G \in\left[D_{j}\right]$ with $G \subset D_{l} \Rightarrow j<l$. Set $X_{j}=\left\{x \in X: D_{x} \in\right.$ $\left.\left[D_{j}\right]\right\}$. Then $X_{j}$ is $D$-invariant, and $\bigcup_{l \geq j} X_{l}$ is closed in $X$ for $1 \leq j \leq t$. One obtains a composition series of $\Gamma_{0}(E)$ by $D$-invariant ideals $I_{j}=\{f \in$ $\Gamma_{o}(E): f$ vanishes on $\left.\bigcup_{l \geq j} X_{l}\right\}$, with subquotients isomorphic to $\Gamma_{o}\left(E_{\mid X_{j}}\right)$, giving rise to a composition series $I_{j} \times_{\hat{\alpha}} D$ of the crossed product $\Gamma_{o}(E) \times_{\hat{\alpha}} D$ with subquotients isomorphic to $\Gamma_{o}\left(E_{\mid X_{j}}\right) \times_{\hat{\alpha}} D$.

Set $Y_{j}=\left\{x \in X_{j}: D_{x}=D_{j}\right\}$, so that $X_{j}=\bigcup_{d \in D} \varphi_{d}\left(Y_{j}\right)$. Given $\tilde{x} \in \widetilde{X}_{j}$, pick a point $x$ in $Y_{j}$ whose image is $\tilde{x}$. By [Ev, Theorem 3.2], there exists a slice at $x$, i.e. a subset $S$ of $Y_{j}$ containing $x$ satisfying

(i) $S$ is closed in $D S=\bigcup_{d \in D} \varphi_{d}(S)$,

(ii) $D S$ is an open neighborhood of the orbit $D x=\bigcup_{d \in D} \varphi_{d}(x)$, so that $\tilde{S}$ is an open neighborhood of $\tilde{x}$,

(iii) $D_{j}$ is the stabilizer subgroup of $y \in S$,

(iv) $d \notin D_{j} \Rightarrow \varphi_{d}(S) \cap S=\varnothing$, and $\widetilde{E_{\mid S}} \cong \widetilde{E_{\mid \widetilde{D S}}}$, where $\widetilde{E_{\mid S}}$ is viewed as a $D_{j}$-bundle. Applying the tilde construction and Corollary 2.6 to $\overline{E_{\mid S}}$ shows that $\widetilde{E_{\mid S}}$ and hence $\widetilde{E_{\overparen{X_{j}}}}$ are locally trivial, and that the fibre $\widetilde{\bar{E}}_{\tilde{y}} \quad($ for $y \in S$ ) is isomorphic to

$$
\left[M_{n} \otimes \mathscr{K}\left(L^{2}(D)\right)\right]^{\alpha^{\prime} \otimes \operatorname{Ad} \rho(D) \mid D_{j}},
$$

which by Lemma 2.4 is again isomorphic to

$$
\begin{aligned}
& {\left[M_{n} \otimes \mathscr{K}\left(L^{2}\left(D_{j}\right)\right)\right]^{\alpha^{y} \otimes \operatorname{Ad} \rho\left(D_{j}\right)} \otimes M_{s_{j}} \cong\left(M_{n} \times_{\alpha^{\prime}} D_{j}\right) \otimes M_{s_{j}},} \\
& s_{j}=\left|D / D_{j}\right|\left(1 \leq s_{j} \leq \infty\right) .
\end{aligned}
$$


By Lemma 2.2, $\widetilde{E_{\widetilde{X_{j}}}}$ decomposes into a countable, disjoint union of locally trivial, homogeneous bundles $\left(E_{j, p}, Z_{j, p}\right)$, where $Z_{j, p}$ is a partition of $\widetilde{X}_{j}$ into clopen sets, and by the sum theorem $\operatorname{dim}\left(\widetilde{X}_{j}\right)=\sup _{p} \operatorname{dim}\left(Z_{j, p}\right)$. If $y \in$ $Z_{j, p}$, then by [Gr, Theorem 18], the fibre $M_{n} \times_{\alpha^{y}} D_{j}$ over $y$ decomposes into a $c_{0}$-sum of matrix algebras with components $M_{n \cdot r}$ contained in $M_{n} \otimes$ $\mathscr{K}\left(L^{2}\left(D_{j}\right)\right)$, where $1 \leq r \leq \sqrt{\left|D_{j}\right|}<\infty$; say the smallest of the components is of the form $M_{n} \otimes M_{r_{j, p}}$. An application of Proposition 2.3 and Nistor's Theorem 7 shows that

$$
\operatorname{sr}\left(\Gamma_{o}\left(E_{j, p}\right)\right)=\left\lceil\frac{\operatorname{dim}\left(Z_{j, p}\right)-1}{2 n r_{j, p} s_{j}}\right\rceil+1 .
$$

Set $l_{j}=\sup _{p} r_{j, p}$ and $v_{j}=\inf _{p} r_{j, p}$. Then,

$$
\begin{aligned}
\left\lceil\frac{\operatorname{dim}\left(\widetilde{X}_{j}\right)-1}{2 n l_{j} s_{j}}\right\rceil+1 & \leq \operatorname{sr}\left(\Gamma_{o}\left(\widetilde{\bar{E}}_{\mid \widetilde{X}_{j}}\right)\right. \\
& =\sup _{p}\left\lceil\frac{\operatorname{dim}\left(Z_{j, p}\right)-1}{2 n r_{j, p} s_{j}}\right\rceil+1 \leq\left\lceil\frac{\operatorname{dim}\left(\widetilde{X}_{j}\right)-1}{2 n v_{j} s_{j}}\right\rceil+1 .
\end{aligned}
$$

By Lemma 2.1, we obtain the following estimate for $\operatorname{sr}\left(\Gamma_{o}(E) \times_{\hat{\alpha}} D\right)$ :

$$
\begin{aligned}
\max _{j}\left\lceil\frac{\operatorname{dim}\left(\widetilde{X}_{j}\right)-1}{2 n l}\right\rceil+1 & \leq \max _{j} \operatorname{sr}\left(\Gamma_{o}\left(\tilde{\bar{E}}_{\widetilde{X}_{j}}\right)\right) \\
& =\operatorname{sr}\left(\Gamma_{o}^{D}(\bar{E})\right) \leq \max _{j}\left\lceil\frac{\operatorname{dim}\left(\widetilde{X_{j}}\right)-1}{2 n s}\right\rceil+1
\end{aligned}
$$

where $l=\max _{j} l_{j} \cdot\left|D / D_{j}\right|$ and $s=\min _{j} v_{j} \cdot\left|D / D_{j}\right|$. Thus,

$$
\operatorname{sr}\left(C_{o}(\tilde{X}) \otimes M_{n \cdot l}\right) \leq \operatorname{sr}\left(\Gamma_{o}^{D}(\bar{E})\right) \leq \operatorname{sr}\left(\Gamma_{o}(E) \otimes M_{s}\right) .
$$

Now if $E$ is an arbitrary bundle, then by (5), (6) and Proposition 2.3, we can find a finite composition series of $\Gamma_{o}(E)$ by $D$-invariant ideals, with subquotients of the form $\Gamma_{o}\left(F_{k}^{q}, Y_{k}^{q}\right)$ for homogeneous $D$-bundles whose fibres are the matrix components $M_{q}$ of $A_{k}$ as in (4), and we obtain a corresponding decomposition series of $\Gamma_{o}^{D}(\bar{E})$ with subquotients $\Gamma_{o}^{D}\left(\bar{F}_{k}^{q}\right) \cong \Gamma_{o}\left(\widetilde{F}_{k}^{q}\right)$. Note that $\operatorname{dim}\left(X_{k}\right)=\operatorname{dim}\left(Y_{k}^{q}\right)$ and consequently, $\operatorname{dim}\left(\widetilde{X_{k}}\right)=\operatorname{dim}\left(\widetilde{Y_{k}^{q}}\right)$ for all $q$. If $l_{k}^{q}$ and $s_{k}^{q}$ are chosen for the bundle $F_{k}^{q}$ as in (10), and if $l=\max _{k, q} q \cdot l_{k}^{q}$ and $s=\min _{k, q} s_{k}^{q}$, then by (10) and Nistor's Theorem 7,

$$
\operatorname{sr}\left(C_{o}(\tilde{X}) \otimes M_{l}\right) \leq \max _{k, q} \operatorname{sr}\left(\Gamma_{o}^{D}\left(\bar{F}_{k}^{q}\right)\right)=\operatorname{sr}\left(\Gamma_{o}^{D}(\bar{E})\right) \leq \operatorname{sr}\left(\Gamma_{o}(E) \otimes M_{s}\right) .
$$

For noncompact $X$, pick a sequence $\left\{T_{m}\right\}_{m=1}^{\infty}$ of $D$-invariant compact sets such that $T_{m} \subset \stackrel{\circ}{T}_{m+1}$ and $X=\bigcup_{m=1}^{\infty} T_{m}$. By the above, there exist $l_{m}$ and 
$s_{m}$ so that $\operatorname{sr}\left(C_{o}\left(\widetilde{T_{m}}\right) \otimes M_{l_{m}}\right) \leq \operatorname{sr}\left(\Gamma_{o}\left(E_{\mid T_{m}}\right) \times_{\hat{\alpha}} D\right) \leq \operatorname{sr}\left(\Gamma_{o}\left(E_{\mid T_{m}}\right) \otimes M_{s_{m}}\right)$. Set $J_{m}=\Gamma_{o}\left(E_{\mid T_{m}}\right) \times_{\hat{\alpha}} D$ and $K_{m}=\Gamma_{o}\left(E_{\mid X-T_{m}}\right) \times_{\hat{\alpha}} D$; then $\Gamma_{o}(E) \times_{\hat{\alpha}} D=\bigcup_{m=1}^{\infty} J_{m}$ and $\Gamma_{o}(E) \times_{\hat{\alpha}} D / K_{m} \cong \Gamma_{o}\left(E_{\mid T_{m}}\right) \times_{\hat{\alpha}} D$, so that by [She, Proposition 3.15],

$$
\operatorname{sr}\left(\Gamma_{o}(E) \times_{\hat{\alpha}} D\right)=\sup _{m} \operatorname{sr}\left(\Gamma_{o}\left(E_{\mid T_{m}}\right) \times_{\hat{\alpha}} D\right) .
$$

Since a similar calculation works for $\operatorname{sr}\left(\Gamma_{o}(E)\right)$ and $\operatorname{sr}\left(C_{o}(\widetilde{X})\right)$, the assertion holds with $l=\sup _{m} l_{m}$ and $s=\inf _{m} s_{m}$.

Remark. The proof of the theorem is valid for an arbitrary second countable compact group $D$ provided that slices exist on all $X_{j}$. This is true for example if all orbits are finite, in which case $\operatorname{dim}(\tilde{X})=\operatorname{dim}(X)$. Inspection of the above process shows that then even $\operatorname{sr}\left(\Gamma_{o}(E) \otimes \mathscr{K}\left(L^{2}(D)\right)\right) \leq \operatorname{sr}\left(\Gamma_{o}(E) \otimes M_{l}\right) \leq$ $\operatorname{sr}\left(\Gamma_{o}(E) \times_{\hat{\alpha}} D\right) \leq \operatorname{sr}\left(\Gamma_{o}(E) \otimes M_{s}\right)$ for some $1 \leq s, l \leq \infty$.

Remark. If $(E, X)$ is a locally trivial, homogeneous $C^{*}$-bundle with fibres $M_{\infty}$, then $\operatorname{sr}\left(\Gamma_{o}(E) \times_{\hat{\alpha}} D\right) \leq \operatorname{sr}\left(\Gamma_{o}(E)\right)$ by a similar proof.

Remark. We can weaken the assumption that the bundle $(E, X)$ have fibres of bounded dimension. In fact, set $X_{n}=\left\{x \in X: \operatorname{dim}\left(E_{x}\right) \leq n\right\}$. Then, $X_{n}$ is $D$-invariant, closed, and $X=\bigcup_{n=1}^{\infty} X_{n}$. Now set $J_{n}=\left\{f \in \Gamma_{o}^{D}(E)\right.$ : $\left.f\left(X-\stackrel{\circ}{X}_{n}\right)=\{0\}\right\}, \quad K_{n}=\left\{f \in \Gamma_{o}^{D}(E): f\left(X_{n}\right)=\{0\}\right\}$, and $J=\overline{\bigcup_{n=1}^{\infty} J_{n}}$. Then, $J_{n}, K_{n}$ and $J$ are closed ideals in $\Gamma_{o}^{D}(E)$. If $\Gamma_{o}^{D}(E)^{+}$denotes the $C^{*}$ algebra with adjoined unit, then by Fell's Stone-Weierstrass theorem, $\Gamma_{o}^{D}(E)^{+} / K_{n}$ $\cong \Gamma_{o}^{D}\left(E_{\mid X_{n}}\right)^{+}$and $\Gamma_{o}^{D}(E)^{+} / J \cong \Gamma_{o}^{D}\left(E_{\mid Z^{0}}\right)^{+}$where $Z^{0}=X-\bigcup_{n=1}^{\infty} \stackrel{\circ}{X}_{n}$. One obtains by [She, Proposition 3.15] that

$$
\operatorname{sr}\left(\Gamma_{o}^{D}(E)\right)=\sup _{n}\left\{\operatorname{sr}\left(\Gamma_{o}^{D}\left(E_{\mid Z^{0}}\right)\right), \operatorname{sr}\left(\Gamma_{o}^{D}\left(E_{\mid X_{n}}\right)\right)\right\} .
$$

The same computation can be done for $\operatorname{sr}\left(\Gamma_{o}^{D}(\bar{E})\right)$ and $\operatorname{sr}\left(\Gamma_{o}(E)\right)$.

(a) If the bundle has fibres of finite dimension locally, i.e. if given $x \in X$, there exists an open neighborhood $U$ of $x$ and an $M$ such that $\operatorname{dim}\left(E_{y}\right) \leq M$ for all $y \in U$, then $x \in \stackrel{\circ}{X}_{M}$. Thus $Z^{0}=\varnothing$, so that the results of this section, in particular (7), (9), and (10) hold.

(b) Set $X^{k}=Z^{k-1}$ and repeat the above construction inductively with $X^{k}$ instead of $X$ and $Z^{k}$ instead of $Z^{0}$. In most nonpathological situations, $Z^{k}=\varnothing$ after a finite number of steps. One can show that $\operatorname{sr}\left(\Gamma_{o}^{D}\left(E_{\mid Z^{k}}\right)\right) \leq$ $\sup _{n} \operatorname{sr}\left(\Gamma_{o}^{D}\left(E_{\mid X_{n}}\right)\right)$ for all $k$, and that similar inequalities apply to $\operatorname{sr}\left(\Gamma_{o}^{D}(\bar{E})\right)$ and $\operatorname{sr}\left(\Gamma_{o}(E)\right)$, so again the results of this section hold. 


\section{REFERENCES}

[Bre] G. E. Bredon, Introduction to compact transformation groups, Academic Press, 1972.

[En] R. Engelking, Dimension theory, North-Holland, 1978.

[Ev] B. Evans, $C^{*}$-bundles and compact transformation groups, Mem. Amer. Math. Soc., no. 269, Amer. Math. Soc., Providence RI, 1982.

[Fe] J. M. G. Fell, The structure of algebras of operator fields, Acta Math. 106 (1961), 233-280.

[Fe1] J. M. G. Fell and R. S. Doran, Representations of $C^{*}$-algabras, locally compact groups, and Banach *-algebraic bundles, vol. 1, Academic Press, 1988.

[Gr] P. Green, The local structure of twisted covariance algebras, Acta Math. 140 (1978), 191-250.

[Le] R. Y. Lee, On the $C^{*}$-algebras of operator fields, Indiana Univ. Math. J. 25 (1976), 303-314.

[Lo] L. H. Loomis, An introduction to abstract harmonic analysis, Van Nostrand, 1953.

[Mo] C. C. Moore, Group extensions and cohomology for locally compact groups III, Trans. Amer. Math. Soc. 221 (1976), 1-33.

[Ni] V. Nistor, Stable rank for a certain class of type I C*-algebras, J. Operator Theory 17 (1987), 365-373.

[Pa] R. S. Palais, Slices and equivariant embeddings, seminar on transformation groups (A. Borel, ed.), Ann. of Math. Studies, no. 46, Princeton Univ. Press, 1960.

[PhR] J. Phillips and I. Raeburn, Crossed products by locally unitary automorphism groups and principal bundles, J. Operator Theory 11 (1984), 215-241.

[Ri] M. Rieffel, Actions of finite groups on $C^{*}$-algebras, Math. Scand. 47 (1980), 157-176.

[Ri2] Dimension and stable rank in the $K$-theory of $C^{*}$-algebras, Proc. London Math. Soc. (3) 46 (1983), 301-333.

[Ro] J. Rosenberg, Some results On cohomology with Borel cochains, with applications to group actions on operator algebras, Operator Theory: Advances and Appl. 17 (1986), 301-330.

[She] A.J.L. Sheu, The cancellation property for modules over the group $C^{*}$-algebras of certain nilpotent Lie groups, Canad. J. Math. 39, 365-427.

[Ta] K. F. Taylor, C*-algebras of crystal groups, Operator Theory: Advances and Appl. 41 (1989), 571-578.

Department of Mathematics, University of Saskatchewan, Saskatoon, S7N 0W0 CANADA 